\title{
Hors série
}

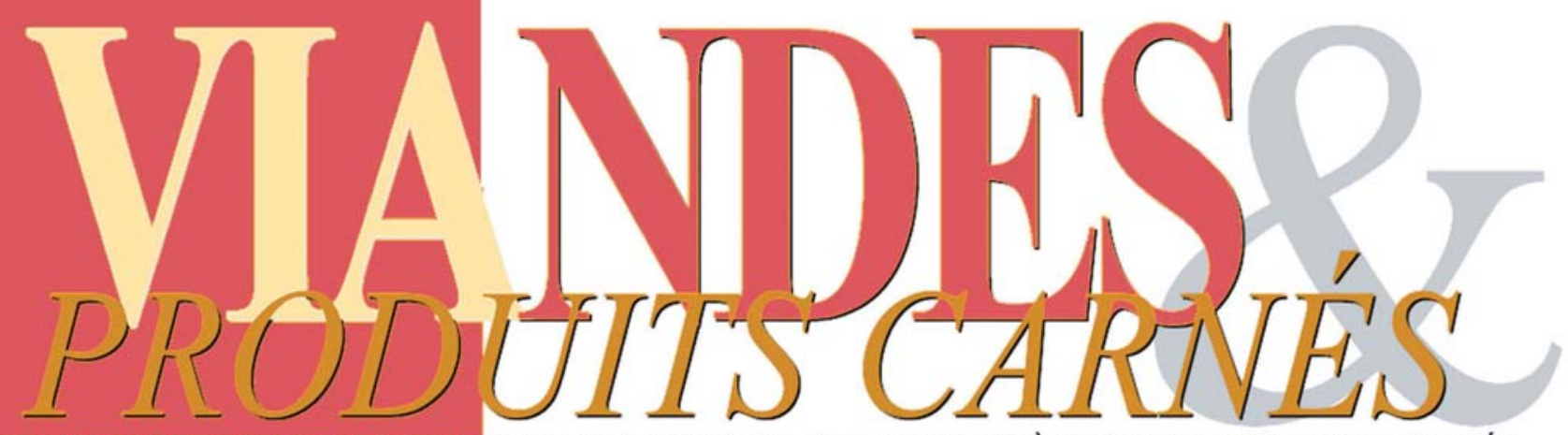

REVUE DES INSTITUTS DE RECHERCHES ET DES CENTRES TECHNIQUES DES FILIĖRES VIANDES ET PRODUITS CARNÉS

\section{$10^{\text {èmes }}$ Journées \\ «Sciences du Muscle et Technologies des Viandes »

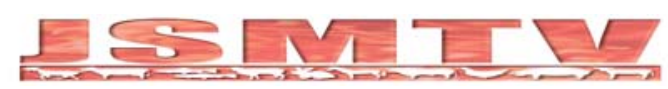

\section{5 et 26 Octobre 2004 RENNES}
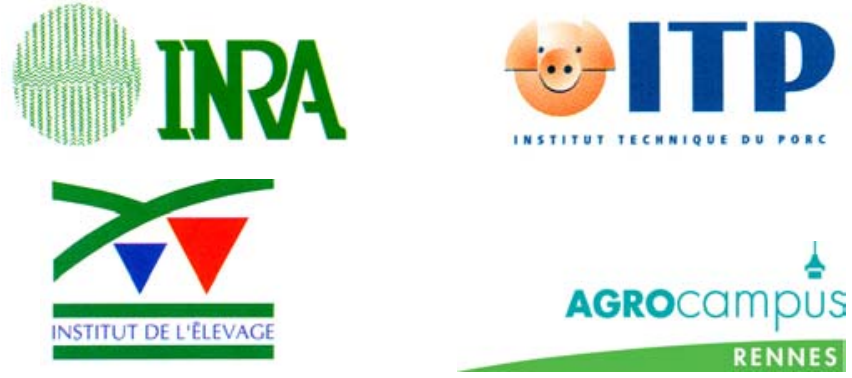


\section{SOMMAIRE}

COMITES D'ORGANISATION

INTRODUCTION

SOMMAIRE

BASE BIOLOGIQUE DE LA QUALITE DE LA VIANDE

SESSION 1

CONFERENCE

Rôle des tissus conjonctifs dans le déterminisme de la tendreté de la viande.

J. LEPETIT

COMMUNiCATIONS ORALES

Variabilité génétique de caractéristiques biologiques du muscle chez des taurillons Charolais.

YOUSSAO A.K.I., RENAND G., PICARD B., JURY C., BERGE P.

Etude de la variabilité de la qualité de la viande de porc par analyse en composantes principales.

LEROY B., ETIENNE G., CHINA B., KORSAK N., DAUBE G., CLINQUART A.

Protéines Sarcoplasmiques et caractérisation protéomique des types musculaires Ovin.

HAMELIN M., SAYD T., CHAMBON C., MONIN G., LAVILLE E.

Influence de l'exercice physique au cours de l'engraissement sur le métabolisme musculaire chez le lapin

GONDRET F., EL RAMOUZ R., FERNANDEZ X., COMBES S.

Influence de l'activité physique chez le lapin : Caractéristique de la carcasse de la viande et

du complexe tendino-osseux.

COMBES S., MOUSSA M., GONDRET F., DOUTRELOUX J.P., REMIGNON H.

Hypertrophie et qualité technologique du filet de poulet

BERRI C., DEBUT M., LE BIHAN-DUVAL E., SANTE-LHOUTELLIER V.,

HAJ HATTAB N., JEHL N., DUCLOS M.J.

Effet des CLA alimentaires sur les performances de croissance et la qualité de la viande de porc.

DOUARD V., MOUROT J., MOUNIER A

\section{Posters}

Caractérisation protéomique de la texture anormalement pâteuse du jambon sec.

SANTE-LHOUTELLIER V., HORTOS M., ARNAU J., MONIN G.

Composition du muscle Longissimus en chaînes lourdes de la myosine entre les races porcines

Large White et Meishan.

LEFAUCHEUR L., ECOLAN P., LE CALLENNEC C.

Effet d'une réversion du sexe sur le développement musculaire du poulet

BAEZA E. , BRILLARD J.P.

Effet du génotype et du gavage sur les dépôts de lipides intramusculaires dans le filet de canard

CHARTRIN P., SCHIAVONE A., BERNADET M.-D., GUY G., MOUROT J., DUCLOS M.J., BAEZA E.

Effet d'un régime à base de glands de chêne vert sur la composition en acides gras des lipides

du tissu musculaire de la cuisse de poulet de chair

BOUDEROUA K. , SELSELET-ATTOU G. , MOUROT J.

Effet du mode d'élevage sur les caractéristiques de la carcasse et celles du muscle du porc local du Bénin.

YOUSSAO A.K.I., MOUROT J. , EDENAKPO., A.ROBIN G., MOUNIER A. 
Catécholamines et relation avec les qualités des viandes de porc : rôles du type génétique, du mode d'élevage et des conditions d'abattage.

BERNE A., ASTRUC T., HAYE E., TERLOUW C.

Qualité nutritionnelle des lipides de viandes : écart liés à l'espèce, écarts liés à l'alimentation :

Quelques observations

CHESNEAU G. , QUEMENER B. , WEILL P.

Effet de différentes teneurs en acide gras n-3 sur la qualité nutritionnelle de la viande de porc.

WILFART A., FERREIRA J., ROBIN G., MOUNIER A., MOUROT J.

Teneur en lipides et métabolisme des adipocytes dans le muscle chez le porc en croissance.

GARDAN D., LOUVEAU I., GONDRET F.

Etudes immunohistologiques de la trame conjonctive de muscles de bovins en relation avec la tendreté.

LABAS R., SIFRE-MAUNIER L., CHANIER L. LISTRAT A., TAYLOR R.

Maturation de la viande de porc : influence sur les mesures de texture. Comparaison Label rouge/Standard.

VAUTIER A., BOULARD J., HOUIX Y., LE ROUX A., MINVIELLE B., BOZEC A.

Caractérisation de l'organisation du tissu conjonctif intramusculaire de la viande bovine par analyse d'images.

SIFRE-MAUNIER L, TAYLOR RG, BERGE P, BONNY JM.

Effets respectifs de la nature de l'alimentation et de la mobilité sur le potentiel métabolique des muscles de bœufs charolais.

JURIE C., ORTIGUES-MARTY I., MICOL D. , CASSAR-MALEK I. , DOZIAS D. ,

PICARD B., HOCQUETTE JF.

Etude de la différenciation des fibres musculaires de l'embryon de truite par hybridation in situ d'un large répertoire de transcrits musculaires.

CHAUVIGNE F., RALLIERE C., CAUTY C., RESCAN P.Y.

Effet de l'âge sur la composition lipidique de la viande d'agneau allaitant italien Merino.

DI CESARE C., FILETTI F., MOUROT J., MAIORANO G., SALVATORI.G., ORIANI G.

Comparaison des tissus adipeux sous-cutanés et intramusculaires chez les porcs Basques et Large White.

L. ALFONSO, J. MOUROT., KIZKITZA INSAUSTI., J.A. MENDIZABAL

Comparaison des qualités de carcasse et de viande du porc Large White et Créole.

RENAUDEAU D., MOUROT J.

Expression des isoformes rapides de chaînes lourdes de myosine dans le muscle pectoralis major de poulet : effet de la sélection pour la croissance et de la nutrition précoce.

BERRI C., HAJ HATTAB N., GODET E., DUCLOS M.J.

QUALITE DE LA CHAIR DE POISSON

SESSION 2

\section{CONFERENCE}

Caractéristiques nutritionnelles des poissons et facteurs de variations.

MEDALE F.

\section{COMMUNICATIONS ORALES}

Evaluation de la spectroscopie de fluorescence frontale pour détecter les poissons congelés/décongelés.

KAROUI R., LARDON S., LAGUET A., THOMAS E., FRENCIA J.P., DUFOUR E.

Taux de sel du saumon fumé et qualité gustative.

FOUCAT L., DONNAT J.P, JOFFRAUD J.J., CARDINAL M., RENOU J.P.

Effet de la nature des huiles sur la fraîcheur et les paramètres de qualité de la chair chez le flétan.

GUILLERM-REGOST C., RØRÅ A.M.B., HAUGEN T., NORVEDT R. 
Remplacement de l'huile de poisson par des huiles végétales dans l'aliment :

incidence sur les mécanismes de constitution des dépôts lipidiques chez la truite arc-en-ciel.

RICHARD N., LARROQUET L., KAUSHIK S.J., CORRAZE G.

\section{Posters}

Distribution du sel dans le saumon fumé en relation avec la distribution du gras :

Etude par imagerie RMN du $1 \mathrm{H}$ et du $23 \mathrm{Na}$..

FOUCAT L., OFSTAD R., DONNAT J.P., RENOU J.P.

Muscles de truite (Onchorhynchus mykiss) fumés conditionnés sous atmosphère modifiée :

Qualité et aspect microbiologique.

QUIÑONES A., CHOUBERT G., GOMEZ R.

Détection des chairs de poisson congelées et décongelées par spectroscopie moyen infrarouge.

GUILLARD A.S., GRONDIN C., LEFUR B., KAROUI R.

Effet d'une supplémentation de l'aliment avec de l'huile de poisson oxydée et de la vitamine E sur la peroxydation lipidique chez des alevins de truite arc-en-ciel

FONTAGNE S., BREQUE J., BISCARRAT B., LINGRAND M., PUYO S.

Remplacement de l'huile de poisson par des huiles végétales chez la truite arc-en-ciel :

Incidence sur les qualités nutritionnelles et organoleptiques.

CORRAZE G., RADUNZ-NETO J., RICHARD N., CARDINAL M. KAUSHIK S.

Effets du jeûne et de la réalimentation sur les caractéristiques du tissu musculaire de truite arc-en-ciel.

LEFEVRE F., PABOEUF G., BUGEON J.

Impact du niveau d'oxygène pendant la croissance sur la structure du muscle et la texture de

la chair de truite (Oncorhynchus mykiss)?

BUGEON J., AUBIN J., PABOEUF G., LEFEVRE F.

Caractérisation des mécanismes protéolytiques dans le muscle de Bar.

CHERET R., DELBARRE-LADRAT C., DE LAMBALLERIE-ANTON M., VERREZ-BAGNIS V.

CRISE ALIMENTAIRE, PRINCIPE DE PRECAUTION ET ECOMIE DES FILIERES

SESSION 3

CONFERENCE

Le principe de précaution : avantages et limites.

DUFOUR B.

Conséquences de l'ESB sur l'industrie de la viande, le témoignage de la SOCOPA

MARIETTE M.

COMMUNICATIONS ORALES

L'équilibre carcasse dans la filière viande bovine française : stratégies et enjeux.

MALOYER-DEVEZE C., JULLIEN M., CAMARET D., LOSSOUARN J.

Les conséquences de l' ESB sur la hausse des prix de détail de la viande de bœuf.

MANSANT P.

La place de la viande bovine dans le modèle alimentaire français : bilan et perspectives.

RAUDE J., CLOUTIER J.

Conséquences des crises de l'ESB sur la segmentation de l'offre en viande bovine :

Exemples de stratégies de la grande distribution en Espagne et en France.

SANS P., DE FONTGUYON G., BRIZ J. 


\section{Posters}

Effets de l'acquisition de l'AOC dans la restauration de la confiance des consommateurs vis-à-vis de la filière bovine : le cas de la Maine Anjou.

LEUSIE M., ALESSANDRIN A

\section{FORMULATION DES PRODUITS}

\section{SESSION 4}

\section{CONFERENCE}

Enjeux de la formulation des préparations et produits à base de viande/ contraintes et solutions.

VENDEUVRE JL. (PAS DE TEXTE)

\section{COMMUNICATIONS ORALES}

Prédiction de la teneur en gras intramusculaire du porc Piétrain par l'ultrasonographie en temps réel.

YOUSSAO A.K.I., VERLEYEN V., MICHAUX C., CLINQUART A., LEROY P. L.

Prédiction du $\mathrm{pH}$ de la viande de dinde au cours du marinage dans des solutions d'acide acétique.

ABI NAKHOUL P., GOLI T., ZAKHIA-ROZIS N., BOHUON P., TRYSTRAM G.

\section{POSTERS}

Effets de différents antioxydants.

GUIAVARC'H E., THOMAS E., PEYRON A., RENERRE M., GATELLIER P., GLADINE C , BAUCHART D., DURAND D.

Diffusion de l'eau dans la viande.

BONNY J-M., MAUNIER L., RENOU J.P.

Quantification des tissus musculaires et adipeux de pièces et de demi carcasses de porc à l'aide de l'imagerie par résonance magnétique.

MONZIOLS M., COLLEWET G., BONNEAU M., MARIETTE F., KOUBA M., DAVENEL A.

\section{SECURITE SANITAIRE DES PRODUITS CARNES \\ SESSION 5}

\section{CONFERENCES}

Recherche de l'origine animale de salmonelloses humaines à Salmonella Typhimurium par caractérisation moléculaire.

CHEMALY M., RIVOAL K., FRAVALO P., ERMEL G., HUMBERT F., SALVAT G.

Maitrise de l'hygiène des viandes bovines

CARTIER P.

\section{COMMUNICATIONS ORALES}

Cinétiques de destruction thermique de Listeria innocua CLIP 20-595

en milieu liquide ou adhérée à des surfaces inertes.

PEROVAL C., SAINT-ANDRE L., PORTANGUEN S., KONDJOYAN A.

Survie de campylobacter jejuni et campylobacter coli sur des échantillons de couenne et de viande de porc stockés à $4^{\circ} \mathrm{C}$

LAROCHE M., KAISER J., FEDERIGHI M., MAGRAS C.

\section{Posters}

Prévalence de la contamination des carcasses de porcs réfrigérées par campylobacter $s p$. Premiers résultats

MIRCOVICH C., LAROCHE M. , DESMONTS M. H., FEDERIGHI M., MAGRAS C. 


\title{
ETUDE DE LA VARIABILITE DE LA QUALITE DE LA VIANDE DE PORC PAR ANALYSE EN COMPOSANTES PRINCIPALES
}

\author{
LEROY B., ETIENNE G., CHINA B., KORSAK N., DAUBE G., CLINQUART A. \\ Département des Sciences des Denrées alimentaires, Faculté de Médecine vétérinaire, \\ Université de Liège, Sart Tilman Bât. B43bis, 4000 Liège (BE).
}

\section{Introduction}

La qualité de la viande de porc dépend de nombreux facteurs tels que le génotype halothane et les conditions pré-abattage (mise à jeun, transport, délai d'attente, etc..). L'étude expérimentale de l'influence de ces facteurs ne prend en compte le plus souvent qu'un seul ou quelques-uns au maximum de ces facteurs alors que, en pratique, c'est l'ensemble de ces facteurs qui détermine la qualité du produit.

L'objectif de ce travail est d'étudier, dans les conditions réelles de production, la variabilité de la qualité technologique, organoleptique et microbiologique de la viande de porc et d'objectiver les relations qui peuvent exister entre les variables évaluées.

\section{Matériel et Méthodes}

Au cours d'une année, 264 porcs ont été abattus au sein de 5 abattoirs de Wallonie. Près de $80 \%$ des porcs provenaient de 4 filières de production porcine distinctes; les 20\% restants étaient issus de la production standard. Le pH45 et la conductivité électrique (Cond45) ont été mesurés $45 \pm 5$ min post mortem dans le muscle Long dorsal entre les apophyses épineuses de la dernière et de l'avant-dernière vertèbre dorsale. Le pH45 a été mesuré au moyen d'une électrode combinée (Ingold réf 104063123) associée à un pH-mètre (Knick 913). La Cond45 a été mesurée à l'aide d'une sonde spécifique associée au P.Q.M. (Pork Quality Meter, Intek). Le poids des abats blancs a été mesuré à l'aide d'une balance électronique suspendue (Kern HCB 20K50). Le poids des carcasses chaudes a été mesuré avant l'étape de refroidissement rapide. Le rapport poids des abats blancs sur poids de carcasse chaude a été calculé afin de rendre compte du respect ou non de la mise à jeun des animaux. Une tranche du muscle Long dorsal, de 2,5 $\mathrm{cm}$ d'épaisseur, a été prélevée $24 \mathrm{~h}$ post mortem afin de mesurer la couleur (CIE L*a*b*), la tendreté (Tend5) et les pertes de jus à la cuisson. La couleur a été mesurée à l'aide d'un spectrocolorimètre Hunter Labscan II qui permet d'objectiver la luminosité ( $\left.\mathrm{L}^{*}\right)$ et la teinte ( $\mathrm{a}^{*}$ et $\left.\mathrm{b}^{*}\right)$. La force maximale appliquée lors du cisaillement d'un échantillon de viande cuite a été mesurée $5 \mathrm{j}$ post mortem au moyen d'une cellule de Warner-Bratzler montée sur un banc de traction Lloyd LR5K. La perte relative de poids après cuisson (50 min, bain-marie à $75^{\circ} \mathrm{C}$ ) a permis d'estimer les pertes de jus à la cuisson. Le génotype halothane a été déterminé par technique PCR selon la méthode décrite par Nakajima et al. (1996). Des écouvillonnages de carcasses ont été réalisés en vue du dénombrement des germes totaux aérobies mésophiles et des E. coli, respectivement selon les méthodes NF-V-08-051 et AFNOR SDP-07/1-07/93 « Rapid E. coli 2 ».

Les relations entre les variables mesurées ont été évaluées par analyse en composantes principales (PCA), analyse multivariée exploratoire qui permet de visualiser de manière synthétique de telles relations. Cette analyse a été effectuée à l'aide du logiciel Statistica.

\section{Résultats et discussion}

Tableau 1 - Variables étudiées + abréviations (Abrév.) utilisées dans les figures 1 et 2 ; nombre d'échantillons ( $n)$; moyenne (Moy.); écart-type (Ecart-t.) ; valeurs minimale (Min.), médiane (Méd.) et maximale (Max.).

\begin{tabular}{|l|l|l|l|l|l|l|l|}
\hline Variables & Abrév. & $n$ & Moy. & $\begin{array}{l}\text { ECART- } \\
\text { T. }\end{array}$ & Min. & Méd. & MAX. \\
\hline Poids carcasse chaude (kg) & PoidsCa & 264 & 93,7 & 9,6 & 64,2 & 93,4 & 122,3 \\
Rapport poids abats blancs / poids carcasse chaude & Rapport & 264 & 0,074 & 0,020 & 0,036 & 0,070 & 0,100 \\
pH 45 min & Ph45 & 264 & 6,02 & 0,35 & 5,30 & 6,07 & 6,80 \\
Conductivité électrique 45 min (mS/cm) & Cond45 & 264 & 5,0 & 2,1 & 3,1 & 4,4 & 22,4 \\
CIE L* (\%) & L $^{*}$ & 264 & 54,3 & 4,5 & 41,6 & 53,5 & 66,4 \\
CIE a* & $a^{*}$ & 264 & 6,3 & 2,0 & 0,9 & 6,2 & 12,3 \\
CIE b* & b $^{*}$ & 264 & 15,5 & 1,8 & 10,5 & 15,4 & 20,1 \\
Force maximale de cisaillement (N) & Tend5 & 264 & 37,1 & 8,2 & 21,0 & 35,8 & 71,7 \\
Pertes de jus à la cuisson (\%) & PerteCui & 264 & 30,0 & 2,6 & 20,2 & 30,0 & 39,2 \\
Germes totaux (ufc/cm $\left.{ }^{2}\right)$ & GermesTo & 264 & 4.236 & 10.762 & 6,7 & 1.266 & 100.000 \\
E.coli $\left(\right.$ ufc/cm $\left.{ }^{2}\right)$ & E.coli & 264 & 11,39 & 34,06 & 0,17 & 1,00 & 366,7 \\
\hline
\end{tabular}


Le tableau 1 présente les 11 variables prises en compte dans la PCA. Il permet de se rendre compte de la variation des paramètres de qualité évalués. Quatre composantes principales d'importance décroissante et non corrélées ont été extraites de cette analyse. Les composantes principales sont des axes perpendiculaires correspondant aux directions dans lesquelles la variabilité est la plus grande. La première composante a permis d'expliquer $28 \%$ de la variabilité ; la composante 2 , $14 \%$; la composante $3,12 \%$ et la quatrième composante, $10 \%$. Ces 4 composantes principales ont permis d'expliquer $63 \%$ de la variabilité totale.

La figure 1 représente les 11 variables initiales dans le plan formé par les axes z1 (composante principale 1) et z2 (composante principale 2). Les coordonnées de ces variables sont les corrélations des variables avec z1 et z2. La composante 1 a présenté une corrélation élevée positive avec le pH45 $(0,80)$; une corrélation négative élevée avec la luminosité $\mathrm{L}^{*}(-0,76)$ et le facteur $\mathrm{b}^{*}(-0,79)$. Une corrélation élevée a été observée entre la composante 2 et $E$.coli. La composante 3 a quant à elle été corrélée avec le rapport poids abats blancs sur poids de carcasse chaude. Enfin, la composante 4 a été fortement corrélée avec la force maximale de cisaillement.

La figure 2 représente, dans le plan formé par z1 et z2, les échantillons classés selon leur génotype halothane. Compte tenu de l'interprétation qui peut être faite du premier axe, les échantillons situés à droite de l'axe zl sont caractérisés par un pH45 élevé et une luminosité $\mathrm{L}^{*}$ faible tandis que le groupe des points situés à gauche de l'axe sont caractérisés par un pH45 faible et une luminosité $\mathrm{L}^{*}$ élevée. De même, les échantillons situés dans la partie négative de l'axe z2 sont caractérisés par un dénombrement des E.coli plus important. Le groupe correspondant aux porcs sensibles au stress, c.-à.-d. de génotype nn, est caractérisé par un pH45 plus faible et une viande plus pâle comparativement au groupe de porcs résistants au stress, c.-à-d. de génotypes $\mathrm{NN}$ et $\mathrm{Nn}$.

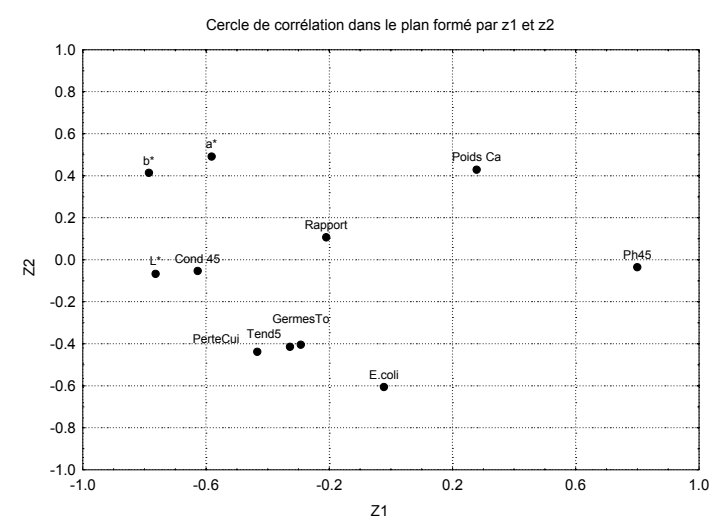

Figure 1 :Représentation des variables relatives à la qualité de la viande dans le plan formé par les $1^{\text {ère }}$ et $2^{\text {ème }}$ composantes principales.

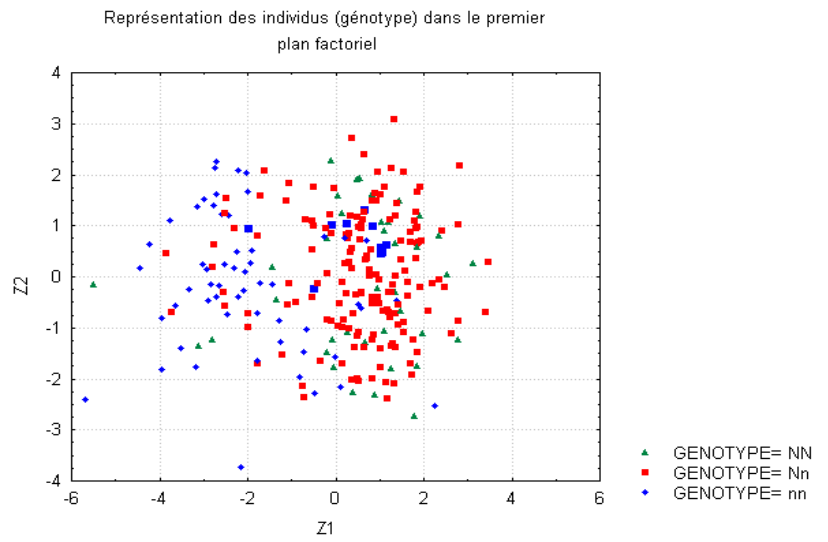

Figure 2 : Représentation des individus dans le plan formé par les $1^{\text {ère }}$ et $2^{\text {ème }}$ composantes principales, en fonction du génotype halothane.

\section{Conclusions}

L'analyse en composantes principales a permis de mettre en évidence des groupes de variables et de visualiser les relations qui peuvent exister entre ces variables. La première composante principale est corrélée aux variables relatives à la qualité technologique et organoleptique de la viande. Elle met en opposition le pH45 d'une part et les paramètres L*, $b^{*}$ et Cond45 d'autre part. La deuxième composante principale peut être considérée comme un axe de qualité microbiologique. L'analyse en composantes principales a également permis de mettre en évidence des groupes d'individus. A titre d'exemple, les génotypes halothane ont pu être différenciés sur ce type de représentation de la variabilité de la qualité de la viande.

\section{Référence bibliographique}

Nakajima E., Matsumoto T., Yamada R., Kawakami K; Tadeka K., Ohnishi A., Komatsu M., 1996. J. Anim. Sci., 74, 29042906.

\section{Remerciements}

Cette étude a été financée par la Direction Qualité des Produits, Direction Générale de l'Agriculture, Ministère de la Région Wallonne, Belgique. 